\title{
Generation of neural progenitors from induced Bama miniature pig pluripotent cells
}

\author{
Xue $\mathrm{Li}^{1,3}$, Zhi-Yan Shan ${ }^{1}$, Yan-Shuang Wu ${ }^{1}$, Xing-Hui Shen ${ }^{1}$, Chun-Jia Liu ${ }^{1}$, Jing-Ling Shen ${ }^{1}$, \\ Zhong-Hua Liu $^{2}$ and Lei Lei ${ }^{1}$ \\ ${ }^{1}$ Department of Histology and Embryology, Harbin Medical University, 194 Xuefu Road, Nangang District, Harbin, \\ Heilongjiang Province, China, ${ }^{2}$ College of Life Science, North East Agricultural University, Harbin, China and \\ ${ }^{3}$ Department of Physiology, Qiqihar Medical University, Qiqihar, China
}

Correspondence should be addressed to Z-Y Shan; Email: shanzhiyan1979@126.com

L Lei; Email: leiys2002@yahoo.com

\begin{abstract}
Pig pluripotent cells may represent an advantageous experimental tool for developing therapeutic application in the human biomedical field. However, it has previously been proven to be difficult to establish from the early embryo and its pluripotency has not been distinctly documented. In recent years, induced pluripotent stem (iPS) cell technology provides a new method of reprogramming somatic cells to pluripotent state. The generation of iPS cells together with or without certain small molecules has become a routine technique. However, the generation of iPS cells from pig embryonic tissues using viral infections together with small molecules has not been reported. Here, we reported the generation of induced pig pluripotent cells (iPPCs) using the iPS technology in combination with valproic acid (VPA). VPA treatment significantly increased the expression of pluripotent genes and played an important role in early reprogramming. We showed that iPPCs resembled pig epiblast cells in their morphology and pluripotent markers, such as OCT4, NANOG, and SSEA1. It had a normal karyotype and could form embryoid bodies, which express three germ layer markers in vitro. In addition, the iPPCs might directly differentiate into neural progenitors after being induced with the retinoic acid and extracellular matrix. Our study established a reasonable method to generate pig pluripotent cells, which might be a new donor cell source for human neural disease therapy.

Reproduction (2014) 147 65-72
\end{abstract}

\section{Introduction}

The miniature pig filled the evolutionary gap with humans because of similarities in organ size, immunology, and whole animal physiology; moreover, it offered several breeding and handling advantages, making it an optimal species for our study (Piedrahita \& Mir 2004, Brandl et al. 2007). Pig pluripotent cells may provide advantages for agricultural purposes to develop disease-free pig productions and biomedical purposes to study human disease mechanisms. Pig pluripotent cells were predominantly derived from the inner cell mass (ICM) in the blastocysts or from the epiblast, which could give rise to all three germ layers of the embryo proper in vivo (Vackova et al. 2007, 2011). However, the establishment of pig embryonic stem cells (ESCs), apart from mouse, human, monkey, and rat, had been proven impossible despite years of maintained effort. There were a number of possible reasons with regard to this problem, containing the choice of wrong stage of embryonic development to establish the cultures, contaminations from rapid differentiation on feeder layers, and, most important, having no optimal culture conditions and identified markers.

Recently, four transcription factors (POU5F1, SOX2, $M Y C$, and KLF4) were shown to be sufficient to reprogram mouse fibroblasts into undifferentiated, pluripotent stem (PS) cells, termed induced PS (iPS) cells (Takahashi \& Yamanaka 2006, Takahashi et al. 2007), which would allow the generation of the most useful model pluripotent cell lines without establishing pig ESCs from the blastocysts or the epiblast. Despite intensive efforts by multiple groups over several years, the characteristics of pig iPS cells, including their morphology, surface markers, and pluripotency, had not been distinctly documented and remain controversial (Esteban et al. 2009, Ezashi et al. 2009, West et al. 2010). Pig iPS cells provide a feasible platform to generate the pluripotent cells 
or germ layer-specific progenitors, which might be useful in differentiating in vitro or the precise genetic engineering in vivo.

Previous studies had shown that chromatin modification was a key step in reprogramming of mouse and human somatic cells. Global gene expression profiling of iPS cells indicated 5-azacytidine (AZA), a DNA methyltransferase inhibitor, and valproic acid (VPA), a histone deacetylases (HDAC) inhibitor, as most potent small molecules in reprogramming of cells in chromatin modification manner (Huangfu et al. 2008, Mikkelsen et al. 2008). It encouraged us to explore whether small molecule could facilitate reprogramming of pig fibroblast cells. Thus, in our paper, we explored the establishment method of pig pluripotent cells from pig fetal fibroblasts by combination of reprogramming factors and small molecules. In addition, the efficiency of the overall process could be affected by the culturing condition, such as culture medium, incubator set, in vitro passage, and so on (Zhao et al. 2010). As the physiological body temperature in pigs was different from that in mice or humans, we detected the effect of culturing temperature on reprogramming, which would yield clues such as how to sustain the growth and pluripotency in vitro and long term.

In vivo study of pig preimplantation development showed that some genes, as pluripotent markers in mouse or human pluripotent cells, displayed different expression patterns in pigs. For example, pig POU5F1 mRNA was expressed in the ICM, epiblast, and trophectoderm (TE) whereas it was not expressed in mouse or human TE. NANOG and SOX2 mRNA were found in all blastomeres during pig preimplantation development until blastocyst formation. In addition, the expression of cell surface markers, SSEA1, SSEA4, TRA-1-60, and TRA-1-81, was discovered both in ICM and in TE of pig blastocyst (Talbot \& Blomberg Le 2008), which suggested that specific markers had not been determined in vivo at the blastocyst stage. In our research, we tried to understand and identify specific markers in maintaining cell pluripotency from induced pig pluripotent cells (iPPCs).

Previous reports described that pig and human neural stem cells (NSCs) appear to be more closely related to each other than mouse NSCs. Moreover, fetal pig neural tissue had been used in several phase 1 clinical trials in patients with Parkinson's or Huntington's disease (Fink et al. 2000), which suggested that the behavior of pig NSCs could well be used in human transplantation study. The generation of pig pluripotent cells would provide a new and effective cell source for inducing the NSCs. In vitro studies had demonstrated that the pig pluripotent cells were able to be differentiated into three germ layers after suspension culture $(\mathrm{Wu}$ et al. 2009), suggesting a potential for multiple lineage differentiation. However, the neural lineage-specific differentiation of pig pluripotent cells had not been reported. In our study, we therefore tested whether neural progenitors could be generated from reprogrammed pig fibroblasts in vitro.

\section{Materials and methods}

\section{Preparation of pig embryonic fibroblasts}

We used Bama miniature pig to isolate pig embryonic fibroblasts (PEFs). About 35-day-old viable fetuses were obtained from the College of Life Science, Northeast Agricultural University, and the skin was minced and exposed to a $0.25 \%$ Trypsin-EDTA (Gibco, Grand Island, NY, USA) solution for $10 \mathrm{~min}$ at $37^{\circ} \mathrm{C}$. After dissociation, cells were washed twice in fresh DMEM (Hyclone) supplemented with $10 \%$ FBS (Invitrogen) and 1\% penicillin and streptomycin (Hyclone, Logan, UT, US), then plated, cultured, and passaged once to obtain first passage of PEFs.

\section{Cell culture}

The PEFs were maintained in DMEM culture medium, only early passages were used for iPPC generation. Mouse embryonic fibroblasts (MEFs) treated with mitomycin C (Sigma) were used as feeder layers. 293T cells were maintained in DMEM culture medium containing 10\% FBS, $2 \mathrm{mM}$ glutamine (Gibco), 1\% nonessential amino acids (Gibco), $1 \mathrm{mM}$ sodium pyruvate (Sigma), and $1 \%$ penicillin and streptomycin. iPPCs were generated and maintained in iPPCs medium, which was knockout DMEM (Gibco) supplemented with $2 \mathrm{mM}$ glutamine, $1 \%$ nonessential amino acids, $0.1 \mathrm{mM}$ 2-mercaptoethanol (Gibco), $10 \mathrm{ng} / \mathrm{ml}$ recombinant human basic fibroblast growth factor (bFGF; Invitrogen) and 20\% knockout serum (Invitrogen), and $2 \mathrm{mM}$ sodium pyruvate, the medium was changed daily. iPPCs were cultured in 37 or $39^{\circ} \mathrm{C}$ in $5 \% \mathrm{CO}_{2}$ incubator respectively.

\section{Retroviral infection and iPPC generation}

293T packaging cells were plated at $8 \times 10^{6}$ cells $/ 100 \mathrm{~mm}$ dish and incubated overnight. Next day, the cells were transfected with pMXs vectors using Lipofectamine LTX and PLUS transfection reagent (Invitrogen). These $\mathrm{pMXs}$ plasmids contain human factors (POU5F1,SOX2, MYC, and KLF4) along with VSV-G envelope (pMD2.G) and packaging vectors (gal-pol), which were kindly provided from Addgene (Cambridge, MA, USA). Twenty-four hours after transfection, the 293T medium was collected as the first virus-containing supernatant and replaced with fresh medium, which was collected after $24 \mathrm{~h}$ as the second virus-containing supernatant. The virus-containing supernatants were filtered through a $0.45 \mu \mathrm{m}$ pore-sized filter and supplemented with $4 \mu \mathrm{g} / \mathrm{ml}$ polybrene. Equal amounts of supernatants containing each of the four retroviruses were mixed, transferred to the fibroblast dish, and incubated overnight. Twenty-four hours after transduction, the viruscontaining medium was replaced with the second supernatant. Next day, the medium was replaced with PEFs medium, small molecules $(0.5 \mathrm{mM}$ AZA, $2 \mathrm{mM}$ VPA, and the mixture of $0.5 \mathrm{mM} A Z A$ and $2 \mathrm{mM}$ VPA) were added to the cells, and 
maintained for 7 days. Eight days after transduction, fibroblasts were harvested by $0.25 \%$ Trypsin-EDTA solution and replated on MEF feeder layer. The medium was changed with iPPCs medium daily. Twenty days after transduction, the colonies were picked up and mechanically dissociated to small clamps by pipetting up and down, and then the cell suspension was transferred on feeder layers in 24-well plates. iPPC colonies were first passaged mechanically using a Pasteur pipette and afterward using $1 \mathrm{mg} / \mathrm{ml}$ collagenase type IV.

\section{Karyotyping}

iPPCs were grown on MEF feeder layers until semi-confluent. Then colchicine was added to a final concentration of $10 \mu \mathrm{g} / \mathrm{ml}$ at $37{ }^{\circ} \mathrm{C}, 5 \% \mathrm{CO}_{2}$ incubator for $1.5 \mathrm{~h}$. Cells were then digested by treating with collagenase IV, pelleted by centrifugation at $800 \mathrm{~g}$ for $5 \mathrm{~min}$, resuspended in $5 \mathrm{ml} 0.075 \mathrm{M} \mathrm{KCl}$ solution, and incubated for $20 \mathrm{~min}$ at $37^{\circ} \mathrm{C}$. Fixative solution composed of one part of acetic acid and three parts of methanol was added to a final volume of $10 \mathrm{ml}$, mixed gently, and incubated for $10 \mathrm{~min}$ at $4{ }^{\circ} \mathrm{C}$. After further centrifugation, the supernatant was removed, and the cells were resuspended in ice-cold fixative solution again. Then the cell suspension was dropped on cold slides. The spreads were stained with Giemsa and 20-30 photographs of separate metaphase karyotypes were counted.

\section{Alkaline phosphatase staining and immunofluorescence}

Alkaline phosphatase (AKP) staining was performed using the Leukocyte Alkaline Phosphatase Kit (Sigma), according to the manufacturer's guidelines. For immunocytochemistry analysis, cells were fixed in $4 \%$ paraformaldehyde for $1 \mathrm{~h}$ at $4{ }^{\circ} \mathrm{C}$. Cells were rinsed with $0.25 \%$ Triton X-100 in DPBS and incubated with $70 \%$ alcohol for $5 \mathrm{~min}$ and then incubated with blocking buffer for $30 \mathrm{~min}$ at room temperature. For antibody staining, cells were followed by incubation with MABs OCT4 (Abcam, Cambridge, UK), NANOG (Abcam), SOX2 (Abcam), SSEA1 (Abcam), SSEA4 (Abcam), TRA-1-60 (Abcam), and TRA-1-81 (Abcam) for $1 \mathrm{~h}$ and then with the appropriate secondary antibodies conjugated to FITC (Santa Cruz) or TRITC (Santa Cruz) for $1 \mathrm{~h}$ at room temperature. After washing three times in DPBS, nuclei were stained with $1 \mu \mathrm{g} / \mathrm{ml}$ Hoechst 33342 (Invitrogen) and visualized under fluorescence microscope (Nikon).

\section{In vitro differentiation}

For embryoid bodies (EBs) formation, iPPCs were harvested by treating with collagenase type IV. The cells were transferred into low attachment plates (Corning, Oneonta, NY, USA) and cultured with differentiation medium, which was similar to the iPPC medium only without bFGF. The medium was changed every other day. Total RNA derived from plated EBs on days 3 or 6 was harvested for RT-PCR analysis. For neural introduction, after 2 days of floating culture, EBs were cultured in differentiation medium supplemented with $2 \mu \mathrm{M}$ all-trans retinoic acid (RA, Sigma) for 4 days. RA-treated EBs were transferred to PLO/laminin-coated plate and cultured in the neural medium supplement with $1 \%$ N2 and B27 for 10 days.

\section{Teratoma}

iPPCs grown on feeder layers were collected by collagenase IV treatment and injected s.c. into NOD-SCID mice. Tumors were observed in 4 weeks after injection, which were collected in 6 weeks and processed for paraffin embedding and hematoxylin and eosin staining.

\section{RT-PCR and real-time PCR analysis}

iPPC and EB cells were collected and total RNA was isolated using TRIzol reagent (Invitrogen). Isolated RNA was used to synthesize cDNA according to instructions from the reverse transcriptase kit (Promega). cDNA samples were normalized based on GAPDH as a reference gene. PCRs were performed using Taq polymerase kit (TransGen Biotech). Single bands of amplification products were confirmed in $2 \%$ agarose gel containing ethidium bromide for visualization. Real-time PCR was performed with TransStart Top Green qPCR SuperMix AQ131-03 (TransGen Biotech) according to manufacturer's instructions. Signals were detected with an CFX96 Real-Time PCR System (Bio-Rad). Primer sequences were listed in Supplementary Table 1, see section on supplementary data given at the end of this article.

\section{Statistical analysis}

All data were obtained from at least three independent experiments. Statistical analysis of the data was performed using Student's $t$-test. $P<0.05$ was considered statistically significant. All data are shown as mean \pm s.D.

\section{Results}

\section{Effects of temperature and small molecules on reprogramming of pig fibroblasts}

Retroviral over-expression of pluripotent gene had been used in pig; however, validated pig iPS cell lines had not been established yet. Considering $39{ }^{\circ} \mathrm{C}$ as the pig physiological body temperature, in our study, we first evaluated the effect of temperature on the early reprogramming of pig iPS cells. PEFs were transfected the four previously described factors (hOCT3/4, hSOX2, $h M Y C$, and $h K L F 4$ ) with the use of retroviral vectors at 37 or $39{ }^{\circ} \mathrm{C}$. Then we performed real-time PCR analysis on the induced PEFs using endogenous pluripotent gene primers for POU5F1, SOX2, NANOG, and KLF4 on day 12 of reprogramming. As shown in Fig. 1, the expression of four pluripotent genes (POU5F1, SOX2, NANOG, and $K L F 4)$ was not significantly different in induced PEFs culturing at $37^{\circ} \mathrm{C}$ compared with those culturing at $39{ }^{\circ} \mathrm{C}(P>0.05)$. Further, we examined the effect of temperature on reprogramming efficiency. ESC-like morphology was used to identify putative iPS colonies, and the reprogramming efficiency was determined by the number of iPS colonies formed out of the total number of infected cells seeded. Of $\sim 1 \times 10^{5}$ infected fibroblasts seeded, 23 iPS colonies were obtained from $39^{\circ} \mathrm{C}$ 

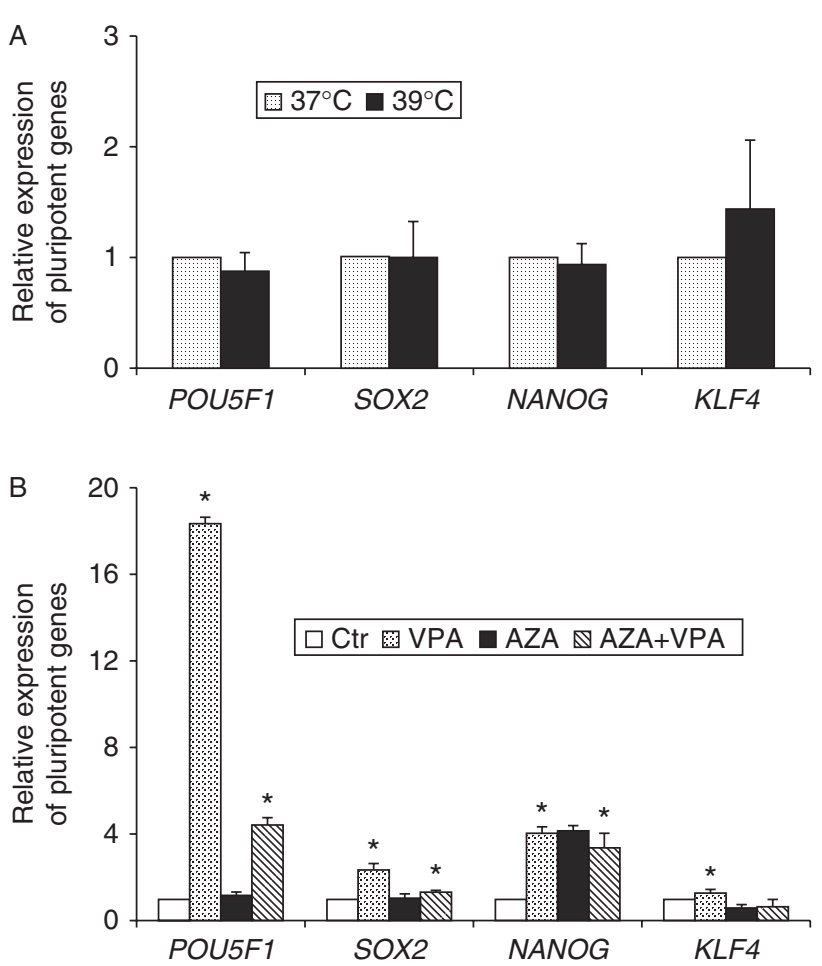

Figure 1 Quantification of mRNA expression of pig pluripotent genes. (A) Comparison of the expression levels of endogenous pluripotent genes in induced cells cultured at 37 and $39{ }^{\circ} \mathrm{C}$. (B) Comparison of the expression levels of endogenous pluripotent genes in induced cells by small molecules. Data shown are representative of results from three independent experiments. Results were normalized to GAPDH expression. ${ }^{*} P<0.01$ (Student's t test, twotailed). AZA, the combination of $A Z A$ and viral infection; $c t r$, four-factor viral infection; VPA, the combination of VPA and viral infection; AZA + VPA, the combination of VPA, AZA, and viral infection.

condition, representing a frequency of $\sim 0.023 \%$, similar to the frequency observed for iPPCs derivation from fibroblasts $(\sim 0.021 \%)$ from $37{ }^{\circ} \mathrm{C}$ condition. This result suggested that the temperature has less effect on the reprogramming of pig fibroblast cells.

Previous studies had shown that small molecules could be beneficial in improving reprogramming efficiency (Huangfu et al. 2008). We tested the effect of known small molecules AZA and VPA on reprogramming of the pig iPPCs. Real-time PCR analysis showed that AZA exhibited no obvious effect on pluripotent genes expression. VPA significantly increased the expression of pluripotent genes, such as POU5F1 ( $>16$-fold change, $P<0.05$ ), SOX2 (greater than equal to twofold change, $P<0.05$ ), and NANOG (greater than equal to fourfold change, $P<0.05)$ compared with control. However, the addition of AZA weakened the increasing roles of VPA on the expression of four transcription factors (Fig. 1B), such as POU5F1 $(<4.5-$ fold difference, $P<0.05)$, SOX2 $(<1.5$-fold difference, $P<0.05)$, and NANOG $(<3.5$-fold difference, $P<0.05)$. Furthermore, of $\sim 1.0 \times 10^{5}$ infected fibroblasts seeded, 25 iPS colonies were obtained in the presence of AZA and 98 colonies were obtained in the presence of VPA. VPA increased the number of ESC-like colonies by fourfold when examined at 1 month post-infection.

It has been suggested that VPA treatment was beneficial to the induction of pig pluripotency whereas AZA was insufficient to reprogram PEFs. This finding implied that histone acetylase was an important epigenetic barrier that PEFs might encounter during reprogramming.

\section{Generation of iPPCs derived from Bama miniature pig fibroblast}

From the results of real-time PCR analysis, we optimized the transduction methods for PEFs as shown in Fig. 2A. The four transcription factors, hOCT3/4, hSOX2, hMYC and hKLF4 were transfected into Bama miniature pig fibroblast with ecotropic retrovirus. Twenty-four hours after transfection, the PEFs were repetitively transfected with the same retrovirus. The next day, the infected cells were supplied with $2 \mathrm{mM}$ VPA (D0) and continued

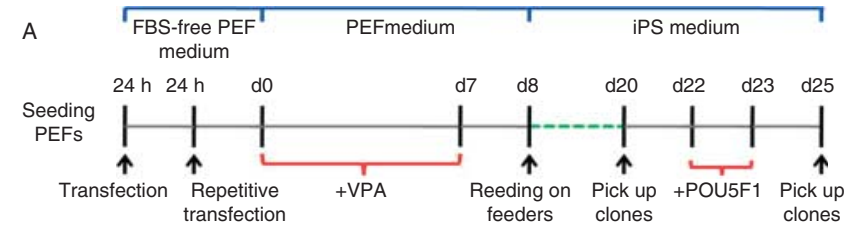

B
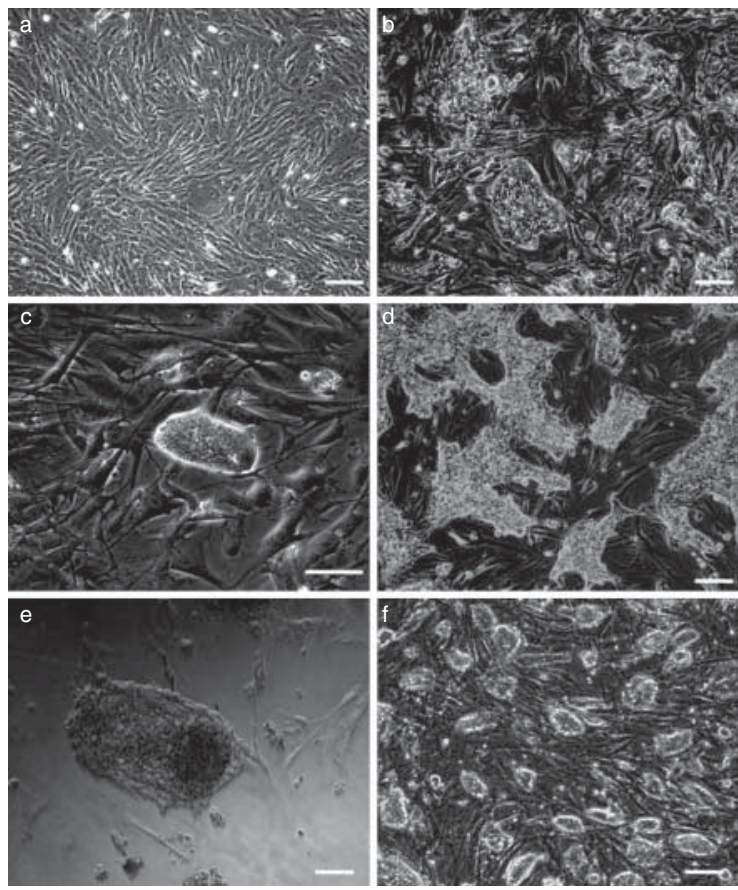

Figure 2 Generation of iPPCs from Bama miniature PEFs. (A) Schematic of the reprogramming protocol used. (B) Sequential morphology of the establishment of iPPCs. (a) PEFs; (b) the morphology of epithelium-like cells after infection at day 12; (c) piPS cell-like colonies formed at day 20; (d) the differentiated piPS cell-like colonies after the third passage; (e) typical compact colonies after repetitive infection; and (f) iPPCs at passage 16. Scale bars, $50 \mu \mathrm{m}$. 
treating for 7 days. On day 8, cells were replated onto feeder layers and the PEF medium was replaced with iPPCs medium. Four days later, some infected cells appeared morphologically different from the parental PEFs, which showed epithelium-like morphology. Approximately 20 days after transduction, we first detected compact colonies comprising reprogrammed PEF cells (Fig. 2). By contrast, no colonies emerged from PEFs transfected with control. ESC-like colonies were handpicked and transferred onto MEFs in the presence of iPPC medium. After passage 3, most of the ESC-like colonies became flat and showed polygonal border. One potential reason for the lack of success was that the reprogramming factors may not provide a continuous supply. Therefore, we tested whether repeated reprogramming factor treatment could yield iPPCs. POU5F1 retrovirus was used to infect the reprogrammed cell secondly. Three days later, the repetitively infected cells gave rise to many compact colonies with clear-cut round edges representing characteristics of mESCs.

\section{Molecular changes occurred in pig fibroblasts reprogramming}

To investigate the molecular changes that occurred during reprogramming, we tested the expression of pluripotent genes in induced cells at different stages by real-time PCR. The expression of pluripotent genes (POU5F1, SOX2, and NANOG) was increased in viral induced cells at day 12 , which suggested that early reprogramming has started. Moreover, the expression level of these genes increased with the extension of time.
However, after three passages, the expression of POU5F1 significantly decreased while that of SOX2 and NANOG reduced slightly. After repetitive infection, the expression of endogenous POU5F1, SOX2, and NANOG was increased; POU5F1 increased dramatically and was maintained at high expression level in long-time culture (Supplementary Figure 1, see section on supplementary data given at the end of this article). Our results showed that early reprogramming was imperfect, which could be completed by repeated infection of reprogramming factor.

\section{Characteristic of reprogrammed pig cells}

The iPPCs generated from the repetitive infection could be readily and stably expanded for long term under conventional human ESC culture conditions (over 30 passages). Each colony showed AKP activity and normal karyotype $(2 n=38)$. Immunofluorescence analysis indicated that iPPCs expressed ESC-specific surface antigens including POU5F1, SOX2, NANOG, Tra-1-60, and Tra-1-81, compared with the negative control (Fig. 3). Interestingly, we detected strong SSEA1 expression in iPPCs and similar observations were reported for pig epiblasts; it suggested that iPPCs maintained epiblastlike characteristics after long-term culture.

To investigate the developmental potential of iPPCs, we used floating cultivation to generate EBs from iPPCs for 7 days. The EBs expressed markers of the three germ layers including GFAP (ectoderm), BMP4 (mesoderm), and AMYLASE (endoderm) as determined by RT-PCR. We injected the iPPCs into NOD-SCID mice and
A

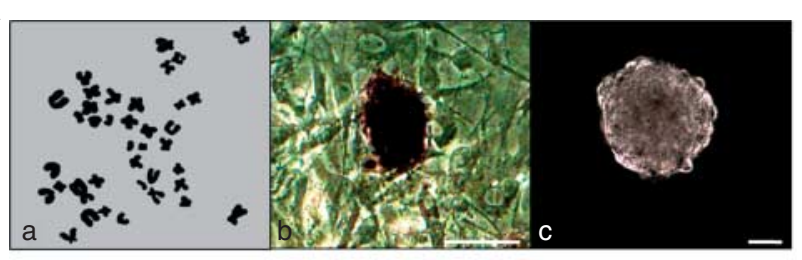

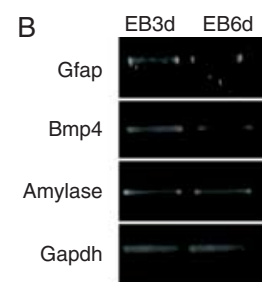

C

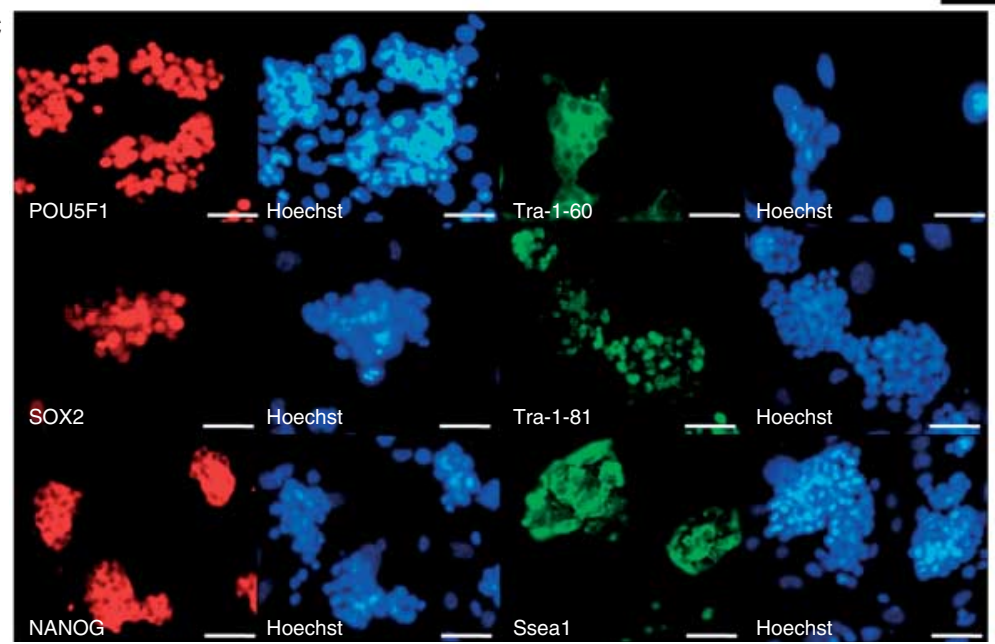

Figure 3 Characterization of iPPCs. (A, a) A normal karyotype (19 pairs) observed in iPPCs; (A, b) alkaline phosphatase-positive colonies; and (A, c) morphology of EBs. (B) RT-PCR analysis of the expression of three germ layer genes in EBs at days 3 or 6 . (C) Immunofluorescence staining of pluripotent gene markers. Scale bars, $50 \mu \mathrm{m}$. 
observed formation of well-encapsulated cystic tumors that harbored differentiated elements of all three germ layers (Fig. 4).

\section{Direct differentiation of iPPCs into neural progenitors}

We then examined whether lineage-directed differentiation of neural progenitors could be induced from the iPPCs. EBs derived from iPPCs were treated with $2 \mathrm{mM}$ RA for 4 days and then were used for immunofluorescence staining. At day 6, Nestin-positive cells were detected in RA-treated EBs; it confirmed that neural differentiation was initiated by RA during EB stage (Fig. 5). Then, these RA-treated EBs were reseeded on PLO/laminin-coated dishes in serum-free culture mediums according to our previous protocol. After 3 days of reseeding, the attached EBs began to extend small branches from the center. At day 10, immunocytochemical staining indicated that the induced cells expressed the neural cell marker TUJ1 (57 $\pm 3 \%)$ and the astrocyte marker GFAP (34 $\pm 2 \%$ ) (Fig. $5 \mathrm{~A}$ and B). This result demonstrated that the iPPCs could be differentiated into neural-like cells, which might provide a useful model in vitro for drug evaluation instead of depending on human cells for neural disease.

\section{Discussion}

As pigs shared physiological similarities with human, the pig pluripotent cells might provide an attractive model to study certain human diseases or assess therapeutical applications in a large animal model (Prather et al. 2003). However, naïve pig pluripotent cells had proven to be particularly difficult to establish from the pig blastocyst (Telugu et al. 2010). Recent studies reported that a widened range of differentiated cells could be converted to iPS cells by introduction of reprogramming factors, which provided a new method to establish pig pluripotential cells (Takahashi \& Yamanaka 2006, Takahashi et al. 2007, Ezashi et al. 2009, Wu et al. 2009). Therefore, in our study, reprogramming procedures developed for human iPS cells establishment had also been adapted to iPPCs.

We first investigated the effect of culture conditions on the early reprogramming of PEFs. The results showed that there was no difference in pluripotent gene expression between 37 and $39{ }^{\circ} \mathrm{C}$ conditions, which suggested that culture temperature might have less effect on the early reprogramming. It is known to all that reprogramming by viral infection was a slow and inefficient process in many species. Previous studies had shown that inhibitors of HDAC or DNA methyltransferase improved the efficiency of reprogramming in mouse and human iPS cells (Huangfu et al. 2008). We speculated that pig reprogramming by defined factors might share common mechanisms with human iPS cells. However, the results showed that AZA alone had no significant improvement on reprogramming whereas VPA treatment significantly increased the expression of pluripotent genes and accelerated the reprogramming. Consistent with previous reports, treatment of AZA showed no effect on PEF reprogramming (Esteban et al. 2009); moreover, the effect of VPA on reprogramming might be due to the collective effects of upregulation of pluripotentspecific genes (Huangfu et al. 2008, West et al. 2010).

Based on the results of the earlier studies, we established a novel and feasible in vitro induction model for iPPCs by combining transcription factors and VPA. The iPPCs showed ESC-like colonies in early establishment; however, the spontaneous differentiation of the peripheral cells was observed after several passages, which might be due to partial reprogramming. A previous study showed that repetitive virus infection could revert the partial reprogramming to a fully reprogrammed state (Warren et al. 2010). Therefore, in our study, the partial reprogramming colonies were repetitively infected with POU5F1. Intriguingly, the endogenous ESCs-related gene expression was dramatically improved accompanied by the increase in numbers of the compact ESC-like colonies. Our results suggested that repetitive infection of POU5F1 guided partially reprogrammed colonies to a fully reprogrammed state, thereby improving the overall reprogramming process. The iPPCs produced colonies of small cells resembling mouse ESCs, which was in accordance with previous delineation about pig epiblast-derived cells grown on STO feeders. Moreover, they expressed epiblast cell markers including POU5F1, SOX2, SSEA-4, TRA-1-60, TRA-1-81, and NANOG protein, especially, SSEA1. This result was consistent with the previous report that carbohydrate antigen SSEA1 was not only expressed on mouse ESCs but also existed weakly in human and pig

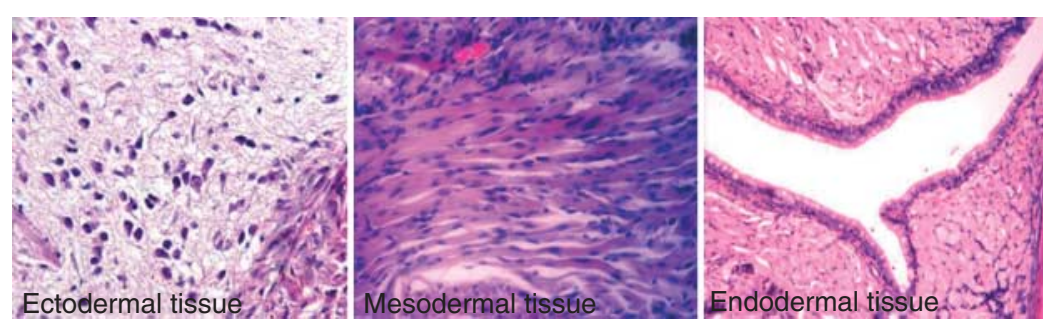

Reproduction (2014) 147 65-72
Figure 4 Xenografts of iPPCs generate teratoma-like masses containing all three embryonic germ layer genes. Hematoxylin and eosin staining shows a teratoma containing multiple tissues. Ectoderm, pigmented retinal epithelium; mesoderm, muscle; and endoderm, respiratory epithelium. 

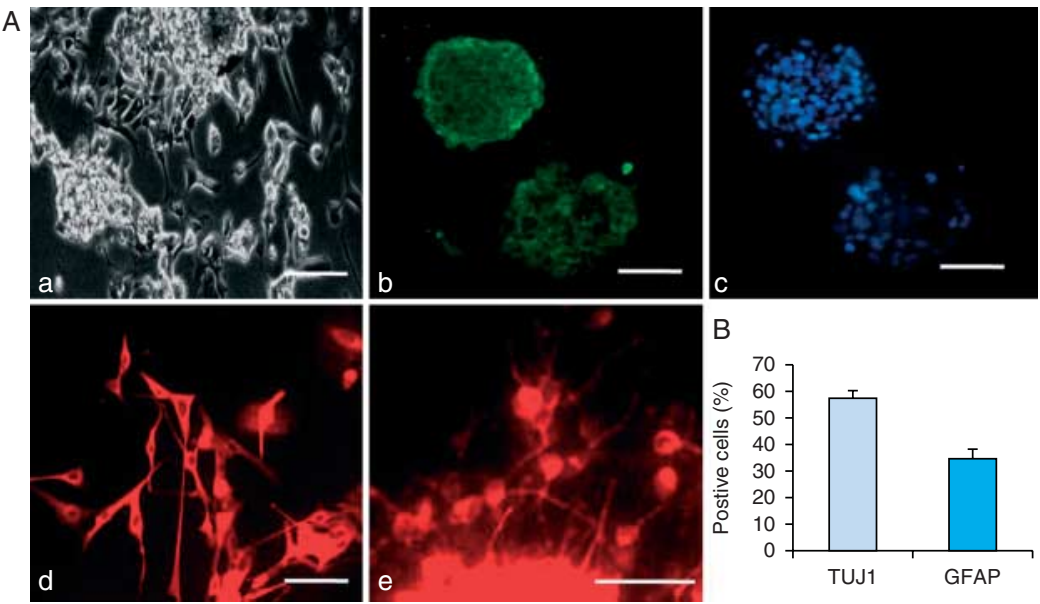

Figure 5 The neural differentiation of iPPCs. (A, a) The neural like cells with long dendrites grown on PLO/laminin-coated dishes; (A, b) NESTIN-positive cells (green); (A, c) staining of nuclei by Hoechst 33342 (blue); (A, d) GFAP-positive cells (red); and (A, e) TUJ1-positive cells (red). Scale bars, $50 \mu \mathrm{m}$. (B) The numbers of TUJ1- or GFAP-positive cells in induced cells derived from RA-induced $\mathrm{EBs}$ on PLO/laminin. epiblast cells (Alberio et al. 2010). In addition, the iPPCs could differentiate into cells representing the three germ layer derivation in vitro. These findings demonstrated that pig pluripotent cells could be generated from fibroblasts of Bama miniature pig by our modified method, which were similar to pig epiblast cells in morphology, surface antigens, and gene expression pattern (Talbot et al. 2002, Hall 2008). Previous researchers reported that the epiblast-like cells had only been maintained in culture for a short time; however, our iPPCs maintaining a normal karyotype have been passaged over 30 passages by now. These results had proved ideal for generating pig pluripotent cells; the long-time maintenance of the pluripotent state needs to be continuously observed in the future.

Although pluripotency of pig iPS cells was demonstrated by teratoma formation and in vitro differentiation as it has been for pig ESCs, pig iPS cells had not produced neural-specific lineage yet. Differentiation of the neural lineage from $\mathrm{mESC}$ demonstrated that high level of RA could be used as an effective inducer of neural progenitor cells (Hayashi et al. 2008, Shan et al. 2011), and extracellular matrix was proved to play an important role in neural-specific differentiation (Shan et al. 2008). Thus, we induced the iPPCs by the combination of RA and laminin/PLO. Our results showed that iPPCs responded to a differentiation protocol for inducing neural differentiation and produced the neuron-specific marker TUJ1 and astrocyte-specific marker GFAPpositive cells. A proper differentiation condition had been established, but further work was still needed to establish the functional neural cells, which might provide a new cell source for clinical transplantation. The critical mechanism of regulation in the divergence of pluripotency to lineage commitment was worthy of further study.

Together, our research had opened a way to generate pluripotent cells for a species in which ESCs had previously proven to be difficult to establish from the early embryo. The analyses of the molecular mechanisms that underlie pig pluripotency and reprogramming might aid in improving the development of pig ESCs. The chromatin of pluripotent cells was more readily reprogrammed than somatic cells, which improve the efficiency of somatic cell nuclear transfer technology that was currently used in the production of genetically engineered ungulates; hence, the establishment of pig iPS cells would be useful in the precise genetic engineering for improved production traits and products.

Furthermore, our induced pluripotent cells could efficiently differentiate into neural cells, which suggest that iPPCs could be induced to differentiate in vitro along prescribed pathways in a reproducible manner, which would be useful for the production of complex biomedical models and provide an alternate source of cells for future cell transplantation.

\section{Supplementary data}

This is linked to the online version of the paper at http://dx.doi. org/10.1530/REP-13-0196.

\section{Declaration of interest}

The authors declare that there is no conflict of interest that could be perceived as prejudicing the impartiality of the research reported.

\section{Funding}

This research is funded by the Postgraduate Innovation Scientific Research Project of Heilongjiang Province of China (YJSCX2011-346HLJ); The National Natural Science Foundation of China (No. 31000645); The National Natural Science Foundation of China (No. 31271590); The State Key Development Program of Basic Research of China (No. 2012CBA01303) and The National Natural Science Heilongjiang Outstanding Youth Fund (No. JC200905). 


\section{Acknowledgements}

The authors thank Dr Na Li for processing the pictures within the manuscript.

\section{References}

Alberio R, Croxall N \& Allegrucci C 2010 Pig epiblast stem cells depend on activin/nodal signaling for pluripotency and self-renewal. Stem Cells and Development 19 1627-1636. (doi:10.1089/scd.2010.0012)

Brandl U, Michel S, Erhardt M, Brenner P, Burdorf L, Jockle H, Bittmann I, Rossle M, Mordstein V, Baschnegger $\mathbf{H}$ et al. 2007 Transgenic animals in experimental xenotransplantation models: orthotopic heart transplantation in the pig-to-baboon model. Transplantation Proceedings 39 577-578. (doi:10.1016/j.transproceed.2006.12.021)

Esteban MA, Xu J, Yang J, Peng M, Qin D, Li W, Jiang Z, Chen J, Deng K, Zhong $\mathbf{M}$ et al. 2009 Generation of induced pluripotent stem cell lines from Tibetan miniature pig. Journal of Biological Chemistry 284 17634-17640. (doi:10.1074/jbc.M109.008938)

Ezashi T, Telugu BP, Alexenko AP, Sachdev S, Sinha S \& Roberts RM 2009 Derivation of induced pluripotent stem cells from pig somatic cells. PNAS 106 10993-10998. (doi:10.1073/pnas.0905284106)

Fink JS, Schumacher JM, Ellias SL, Palmer EP, Saint-Hilaire M, Shannon K, Penn R, Starr P, VanHorne C, Kott HS et al. 2000 Porcine xenografts in Parkinson's disease and Huntington's disease patients: preliminary results. Cell Transplantation 9 273-278. (doi:10.1115/1.2837074)

Hall V 2008 Porcine embryonic stem cells: a possible source for cell replacement therapy. Stem Cell Reviews 4 275-282. (doi:10.1007/ s12015-008-9040-2)

Hayashi K, Lopes SM, Tang F \& Surani MA 2008 Dynamic equilibrium and heterogeneity of mouse pluripotent stem cells with distinct functional and epigenetic states. Cell Stem Cell 3 391-401. (doi:10.1016/j.stem. 2008.07.027)

Huangfu D, Maehr R, Guo W, Eijkelenboom A, Snitow M, Chen AE \& Melton DA 2008 Induction of pluripotent stem cells by defined factors is greatly improved by small-molecule compounds. Nature Biotechnology 26 795-797. (doi:10.1038/nbt1418)

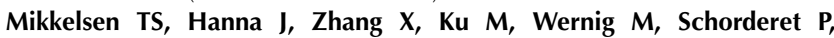
Bernstein BE, Jaenisch R, Lander ES \& Meissner A 2008 Dissecting direct reprogramming through integrative genomic analysis. Nature $\mathbf{4 5 4}$ 49-55. (doi:10.1038/nature07056)

Piedrahita JA \& Mir B 2004 Cloning and transgenesis in mammals: implications for xenotransplantation. American Journal of Transplantation 4 (Suppl 6) 43-50. (doi:10.1111/j.1600-6135.2004.0344.x)

Prather RS, Hawley RJ, Carter DB, Lai L \& Greenstein JL 2003 Transgenic swine for biomedicine and agriculture. Theriogenology 59 115-123. (doi:10.1016/S0093-691X(02)01263-3)

Shan ZY, Shen JL, Li QM, Wang Y, Huang XY, Guo TY, Liu HW, Lei L \& Jin LH 2008 pCREB is involved in neural induction of mouse embryonic stem cells by RA. Anatamoical Record 291 519-526. (doi:10.1002/ ar.20686)
Shan ZY, Liu F, Lei L, Li QM, Jin LH, Wu YS, Li X \& Shen JL 2011 Generation of dorsal spinal cord GABAergic neurons from mouse embryonic stem cells. Cellular Reprogramming 13 85-91. (doi:10.1089/cell.2010.0055)

Takahashi K \& Yamanaka S 2006 Induction of pluripotent stem cells from mouse embryonic and adult fibroblast cultures by defined factors. Cell 126 663-676. (doi:10.1016/j.cell.2006.07.024)

Takahashi K, Tanabe K, Ohnuki M, Narita M, Ichisaka T, Tomoda K \& Yamanaka S 2007 Induction of pluripotent stem cells from adult human fibroblasts by defined factors. Cell 131 861-872. (doi:10.1016/j.cell. 2007.11.019)

Talbot NC \& Blomberg Le A 2008 The pursuit of ES cell lines of domesticated ungulates. Stem Cell Reviews 4 235-254. (doi:10.1007/ s12015-008-9026-0)

Talbot NC, Powell AM \& Garrett WM 2002 Spontaneous differentiation of porcine and bovine embryonic stem cells (epiblast) into astrocytes or neurons. In Vitro Cellular \& Developmental Biology. Animal 38 191-197. (doi:10.1290/1071-2690(2002)038<0191:SDOPAB > 2.0.CO;2)

Telugu BP, Ezashi T \& Roberts RM 2010 Porcine induced pluripotent stem cells analogous to naive and primed embryonic stem cells of the mouse. International Journal of Developmental Biology 54 1703-1711. (doi:10.1387/ijdb.103200bt)

Vackova I, Ungrova A \& Lopes F 2007 Putative embryonic stem cell lines from pig embryos. Journal of Reproduction and Development $\mathbf{5 3}$ 1137-1149. (doi:10.1262/jrd.19108)

Vackova I, Novakova Z, Krylov V, Okada K, Kott T, Fulka H \& Motlik J 2011 Analysis of marker expression in porcine cell lines derived from blastocysts produced in vitro and in vivo. Journal of Reproduction and Development 57 594-603. (doi:10.1262/jrd.10-184H)

Warren L, Manos PD, Ahfeldt T, Loh YH, Li H, Lau F, Ebina W, Mandal PK, Smith ZD, Meissner A et al. 2010 Highly efficient reprogramming to pluripotency and directed differentiation of human cells with synthetic modified mRNA. Cell Stem Cell 7 618-630. (doi:10.1016/j.stem.2010. 08.012)

West FD, Terlouw SL, Kwon DJ, Mumaw JL, Dhara SK, Hasneen K, Dobrinsky JR \& Stice SL 2010 Porcine induced pluripotent stem cells produce chimeric offspring. Stem Cells and Development 19 1211-1220. (doi:10.1089/scd.2009.0458)

Wu Z, Chen J, Ren J, Bao L, Liao J, Cui C, Rao L, Li H, Gu Y, Dai H et al. 2009 Generation of pig induced pluripotent stem cells with a drug-inducible system. Journal of Molecular Cell Biology 1 46-54. (doi:10.1093/jmcb/mjp003)

Zhao XY, Li W, Lv Z, Liu L, Tong M, Hai T, Hao J, Guo CL, Wang X, Wang L et al. 2010 Efficient and rapid generation of induced pluripotent stem cells using an alternative culture medium. Cell Research 20 383-386. (doi:10.1038/cr.2010.26)

Received 16 May 2013

First decision 3 June 2013

Revised manuscript received 9 October 2013

Accepted 14 October 2013 